\title{
Application of Audio Computer-Assisted Self-Interviews to Collect Self-Reported Health Data: An Overview
}

\author{
J.L. Brown ${ }^{a} \quad$ A. Swartzendruber ${ }^{\text {b }} \quad$ R.J. DiClemente ${ }^{b}$ \\ ${ }^{a}$ Department of Psychology, Texas Tech University, Lubbock, Tex. and ${ }^{b}$ Rollins School of Public Health, \\ Emory University, Atlanta, Ga., USA
}

\section{Key Words}

Audio computer-assisted self-interviews · Review ·

Self-report

\begin{abstract}
For assessment of sensitive health behaviors (e.g., sexual behavior, violent behaviors, substance use), research is typically limited to an examination of self-reports of past behavior. Audio computer-assisted self-interviews (ACASI) may enhance the validity of self-report data in research and clinical settings by reducing measurement bias. This paper provides an introduction to ACASI for collection of self-reported health data. The potential benefits and cost-effectiveness of ACASI use in research and clinical settings are reviewed. We then review the theoretical underpinnings that may underlie differential reporting of health behaviors between assessment modalities. Next, we highlight studies that have investigated differences in self-reported health behaviors between assessment modalities. Lastly, we summarize potential applications of ACASI assessments within clinical settings.
\end{abstract}

(c) 2013 S. Karger AG, Basel

Research that informs health behavior change interventions and health policy involves many challenges for investigators, including the challenge of accurately as- sessing the health practices of those who are at greatest risk for adverse health outcomes. For assessment of sensitive health behaviors (e.g., substance use), research is typically limited to an examination of self-reports of past behavior. Self-reports of sensitive health behaviors are sometimes influenced by motivational biases [Schroder et al., 2003]. As such, assessment approaches that enhance the validity of self-report data are essential in health research and clinical settings.

In recent years, audio computer-assisted self-interviews (ACASI) have grown in popularity as an alternative to paper and pencil self-administered questionnaires (SAQ) and interviewer-administered questionnaires (IAQ) for collecting self-report data in psychological and behavioral research. A desire to reduce bias in the measurement of behaviors associated with HIV transmission provided motivation for the development of ACASI technology in the 1990s [Harmon et al., 2009]. ACASI has been most widely adopted for studies or clinical settings involving assessment of sensitive behaviors or with stigmatized populations, including assessment of sexual risk behavior [Romer et al., 1997; Des Jarlais et al., 1999; Johnson et al., 2001; Kurth et al., 2004; Brown and Vanable, 2009], psychiatric symptoms [Epstein et al., 2001; Chinman et al., 2004], and substance use [Islam et al., 2012]. In addition, ACASI has been used in experimental research to study cognitive functioning [Günther et al.,

\section{KARGER}

E-Mail karger@karger.com

www.karger.com/cre
(C) 2013 S. Karger AG, Basel

0008-6568/13/0477-0040\$38.00/0
Jennifer L. Brown, PhD

Department of Psychology, Texas Tech University MS 42051

Lubbock, TX 79409 (USA)

E-Mail jennifer.brown@ttu.edu 
2003; Blais et al., 2005], the efficacy of computer-based instruction [Cumming and Elkins, 1996], and to conduct neuropsychological assessments [Davidson et al., 1987; White et al., 2003]. Indeed, ACASI has great potential to assess a range of self-report domains and for a multitude of clinical settings and experimental designs. Dental selfreport items have been validated for assessment of oral health and show good potential for the assessment of periodontal disease [Jamieson et al., 2004; Blicher et al., 2005]. Further, dental researchers and clinicians commonly collect self-reported behaviors, attitudes and experiences. However, we are aware of few dental research studies which have utilized this technology.

This paper provides an introduction to the use of ACASI for collection of self-reported health behavior data. In what follows, we first provide an overview of ACASI. Next, we describe the potential benefits and costeffectiveness of ACASI in research and clinical settings. We then review the theoretical underpinnings that may underlie differential reporting of health behaviors between various assessment modalities. Next, we highlight studies that have investigated differences in self-reported sensitive health behaviors between assessment modalities. Lastly, we summarize potential applications of ACASI assessments within clinical settings.

\section{ACASI Overview}

ACASI presents individual questions visually to the participant on a computer screen. Through headphones, respondents listen to the questions as they are presented via digitally generated or recorded audio voice-overs. The respondent enters responses by using a touch screen, mouse or keyboard. ACASI assessments can also be administered using telephones with the telephone keypad serving as the input device. ACASI digitally records participants' responses, and the data are easily exported to most statistical software packages. Implementation of ACASI requires either a desktop or laptop computer and the purchase of ACASI computer software. A number of ACASI software packages are currently available on the market (e.g., Questionnaire Design Studio, BLAISE, MediaLab). These software packages provide considerable flexibility in the design and presentation of questionnaires and each offers unique features. ACASI software programs often utilize user-friendly menus to program survey items, select question types, and add additional features.

Application of ACASI
Table 1. Overview of benefits of assessment modalities

\begin{tabular}{llll}
\hline & ACASI IAQ SAQ \\
\hline 1. Self-administered & $\checkmark$ & & $\checkmark$ \\
2. Reduces literacy concerns & $\checkmark$ & $\checkmark$ & \\
3. Increased cost-effectiveness & $\checkmark$ & & \\
4. Reduced staff requirements & $\checkmark$ & & $\checkmark$ \\
5. Decreased motivational biases to & $\checkmark$ & & $\checkmark$ \\
$\quad$ underreport sensitive health behaviors & & & \\
6. Facilitates complex survey administra- \\
$\quad$ & $\checkmark$ & $\checkmark$ & \\
tion patterns, range rules, consistency & & & \\
checks & & & \\
7. Decreased data entry errors & & & \\
\hline
\end{tabular}

\section{Benefits of ACASI}

In recent years, ACASI has received increased attention and use, as it affords a number of potential benefits for researchers, clinicians and respondents (table 1). For complex surveys, computerized assessment modes reduce the burden of survey completion through the use of automatic branching, range rules, and consistency checks [Erdman et al., 1985; Turner et al., 1998; Metzger et al., 2000; Schroder et al., 2003]. In low-literacy populations or for multilingual samples, ACASI ameliorates literacy concerns that may affect data quality in alternate assessment modes, such as SAQs [Turner et al., 1998; Schroder et al., 2003; Perlis et al., 2004]. Additionally, ACASI decreases the number of staff hours devoted to interviewing and data entry and verification [Jennings et al., 2002]. ACASI may also improve data accuracy by reducing data entry errors [Metzger et al., 2000].

ACASI may minimize missing data through automatic branching and the privacy it provides to respondents. It may also lessen nonresponse rates given that the technology allows for visual design of the survey and embedding of pictures, symbols and videos within the survey, which may help to keep the respondent engaged in survey completion. These visuals may also be used to educate respondents or visually supplement instructions or questions in a standard fashion.

The use of IAQ for collecting self-reported data on sensitive behaviors has been criticized because of concerns about participant self-presentation biases and interviewer biases [Turner et al., 1998]. In comparison with IAQs, ACASI removes possible interviewer bias that may affect responses [Metzger et al., 2000]. Additional interviewer characteristics, including speed by which ques- 
tions are asked, body language, and personal characteristics such as race, gender, and age are eliminated through use of computer assessments [Bloom, 1998]. Furthermore, for large-scale projects, ACASI may reduce the error variance associated with using multiple interviewers [Bloom, 1998]. Thus, use of ACASI may lessen bias concerns often associated with the use of IAQ for collecting self-report data on sensitive sexual behaviors.

Relative to IAQ, SAQ provides increased privacy and may reduce motivational bias to report in a socially desirable fashion. ACASI may further enhance the perception that information remains confidential relative to SAQ because individual responses are not easily viewed by research or clinical staff. As a result, ACASI may reduce the amount of embarrassment or discomfort an individual experiences when disclosing sensitive information (e.g., sexual behavior, illicit drug use) and may result in reduced motivational bias to self-report in a socially desirable manner [Erdman et al., 1985]. This effect may be magnified by the perceived sensitivity of the behavior assessed.

\section{Cost-Effectiveness of ACASI}

While ACASI offers a number of potential benefits to researchers, clinicians, and respondents, the cost of the ACASI software and computer hardware needed may preclude its use in some research and clinical settings. Costs for the purchase of ACASI software range from several hundred dollars to as a high as thousands of dollars for a single license (depending on software sophistication and licensing specifics) and computer hardware purchases add additional expense. On the other hand, ACASIbased data collection may yield considerable cost savings over time for some studies or settings because of increased efficiency, reduced survey duplication costs, and the elimination of staff time devoted to data entry and survey administration. Brown et al. [2008] conducted a comparative cost analysis to evaluate the difference in costs between ACASI and SAQ assessment types to determine the relative expenses for each approach with varying parameter considerations [Levin, 1983; Brown et al., 2008]. Such an analysis allows researchers and practitioners to compare the initial fixed costs and the variable administrative costs associated with a given assessment type.

To facilitate researchers' and practitioners' choice between ACASI and SAQ, this study provided theoretical cost models with specific parameters to compare the costs for each assessment type [Brown et al., 2008]. Utilizing these cost models, the study compared the cost-effectiveness in a health behavior study where both ACASI and SAQ questionnaires were administered. Given the high initial costs, ACASI was found to be less cost-effective than SAQ for a single study. However, the projected cost models that manipulated specific study parameters suggested that in a variety of other research or clinical settings, ACASI was a more economical choice. Data from these models indicated several conditions where ACASI was more cost-effective than SAQ. Indeed, ACASI is particularly cost-effective for studies or clinics assessing a large number of individuals, as staff costs for SAQ and IAQ administration and data entry are eliminated. In addition, when computer and ACASI software purchases are to be used for multiple studies or for long-term clinical usage, ACASI will be the most cost-effective assessment modality because the impact of the high initial fixed costs are distributed across multiple investigations or over time.

\section{Differential Disclosure of Sensitive Health Behaviors by Assessment Mode: Theoretical Underpinnings}

The accuracy of self-report data is influenced by a variety of factors, including the cognitive demands of the recall task and motivational biases [Catania et al., 1990; Turner et al., 1997; Schaeffer, 2000; Tourangeau, 2000; Schroder et al., 2003]. Motivational biases may lead individuals to distort their self-reports of past behavior to avoid shame or embarrassment, or to appear in a more favorable light [Catania et al., 1990; Swadi, 1990; Turner et al., 1997]. Motivational biases may be particularly pertinent to reporting of sensitive health behaviors, where it is commonly assumed that individuals underreport risk behaviors because of the sensitive, personal, and sometimes stigmatizing nature of such behaviors [Catania et al., 1990; Turner et al., 1997; Weinhardt et al., 1998]. The use of ACASI may provide reassurance that responses are truly private, as data entered via computer are not easy to review (e.g., by research or clinic staff) relative to written information provided through paper and pencil surveys. In turn, the perception that ACASI responses are more anonymous may reduce socially desirable responding, an effect that may be magnified when responding to socially sensitive questions.

It has been hypothesized that ACASI-based assessments reduce the motivational bias to self-report in a socially desirable fashion by providing a greater degree of privacy than other assessment modes such as IAQ and 
SAQ [Paperny et al., 1990; Kurth et al., 2001]. Specifically, the level of confidentiality and anonymity provided by an assessment type may result in differences in self-reported behaviors [Turner et al., 1997]. Anonymity is defined as providing no identifying information or means by which to identify the identity of the respondent. With confidential assessments, identifying information may be collected, but is protected by the researcher or clinician, such that individual identities are not identified in data analyses, publication or presentation of findings, or shared with individuals outside of the research or clinical context. Thus, individuals may perceive ACASI to be more anonymous in nature because of the inability to immediately review responses. Therefore, motivational bias to underreport sensitive behaviors may be further reduced when assessed under more private, anonymous conditions using an ACASI-delivered assessment.

\section{Differential Disclosure of Sensitive Health Behaviors by Assessment Mode}

Assessment mode refers to the method by which survey questions are presented to gather self-report data. A growing number of studies have compared rates of selfreported sensitive health behaviors between assessment modes. Such studies assume that higher reports of sensitive health behaviors are indicative of increased validity because of individuals' tendency to underreport risk behaviors [Catania et al., 1990; Turner et al., 1997; Weinhardt et al., 1998; Brener et al., 2003]. In what follows, we summarize studies that have examined differences in selfreported sensitive health behaviors between: (a) ACASI and IAQ, and (b) ACASI and SAQ.

Self-Reports of Sensitive Behaviors: ACASI versus IAQ Computerized assessments typically result in higher frequency reports of sensitive behaviors relative to IAQ which afford participants minimal levels of privacy and, therefore, may result in greater motivational biases [Schroder et al., 2003]. For example, in studies of adult populations identified as being at elevated risk for HIV, such as in samples of men who have sex with men, intravenous drug users, and sexually transmitted disease clinic patients, higher frequencies of risk behaviors are reported in ACASI conditions when compared to data obtained through IAQ [Des Jarlais et al., 1999; Metzger et al., 2000; Newman et al., 2002; Kurth et al., 2004]. For instance, in a study among youth, a greater proportion reported smoking via telephone ACASI relative to IAQ conducted over the telephone, especially among females, and particularly those who reported their parents would strongly disapprove of their smoking [Currivan et al., 2004]. In some research settings ACASI has not been shown to be superior to IAQ. For example, in one study, internal reliability and mean scores from standardized symptom surveys were similar between ACASI and IAQ assessments conducted $20 \mathrm{~min}$ apart for patients with mental illness [Chinman et al., 2007].

\section{Self-Reports of Sensitive Behaviors: ACASI versus SAQ}

The vast majority of studies which have assessed differences in self-reports via ACASI relative to SAQ have assessed substance use and sexual behaviors. A number of reports indicate that ACASI yields higher reports of sensitive behaviors (i.e., illicit drug use and sexual risk behavior) relative to data derived from SAQ [Robinson and West, 1992; Turner et al., 1998]. However, findings have varied across study populations and behaviors assessed. When comparing self-reports of sensitive behaviors between ACASI and SAQ, two studies have found greater disclosure of sensitive behaviors with ACASI [Robinson and West, 1992; Turner et al., 1998]. However, three other studies have found inconsistent patterns of self-reports or nonsignificant differences between the two assessment types [Webb et al., 1999; Johnson et al., 2001; Brown and Vanable, 2009]. Conclusive evidence is lacking for the increased accuracy of self-reported HIV-risk behaviors and substance use when collected via ACASI when compared to SAQ. As these assessment types are both self-administered, the effects of privacy level and associated motivational biases for disclosure of sensitive information may be similar for ACASI and SAQ.

\section{Potential Applications of ACASI to Clinical Settings}

ACASI programs have been used for screening in clinical settings [Williams et al., 2004; Chisolm et al., 2009] and shown to improve care for a variety of diseases, including diabetes and asthma [Cherry et al., 2002; Guendelman et al., 2002]. ACASI has potential to broaden these applications, and several studies have assessed the feasibility of ACASI within clinical settings. For example, ACASI screening prior to scheduled HIV clinical care visits was found to be feasible and acceptable to both patients and health providers and useful for identifying patients with inadequate medication adherence and symptoms of depression [Schackman et al., 2009]. Further, ACASI surveys on symptoms, drug use, medication adherence and 
side effects completed by patients in mental health clinics awaiting their appointments was found to be feasible and enjoyable to patients. While health care providers reported the information gained resulted in little impact on their care, specific suggestions for improvement were obtained [Chinman et al., 2007].

\section{Conclusions}

ACASI has several advantages over IAQ and SAQ in terms of ease of data collection. ACASI may also be more cost-effective and yield more accurate data and lower nonresponse rates. Although ACASI has not commonly been used in dental research or practice, studies in other areas show that ACASI has useful applications in both research and clinical settings.

\section{Acknowledgments}

Jennifer L. Brown was supported by K12 GM000680 from the National Institute of General Medical Sciences.

\section{Disclosure Statement}

None of the authors have any conflict of interest to disclose.

\section{References}

Blais AR, Thompson MM, Baranski JV: Individual differences in decision processing and confidence judgments in comparative judgment tasks: the role of cognitive styles. Pers Individ Dif 2005;38:1701-1713.

Blicher B, Joshipura K, Eke P: Validation of selfreported periodontal disease: a systematic review. J Dent Res 2005;84:881-890.

Bloom DE: Technology, experimentation, and the quality of survey data. Science 1998;280:847848.

Brener ND, Billy JO, Grady WR: Assessment of factors affecting the validity of self-reported health-risk behavior among adolescents: evidence from the scientific literature. J Adolesc Health 2003:33:436-457.

Brown JL, Vanable PA: The effects of assessment mode and privacy level on self-reports of risky sexual behaviors and substance use among young women. J Appl Soc Psychol 2009;39: 2756-2778.

- Brown JL, Vanable PA, Eriksen MD: Computer assisted self-interviews: a cost effectiveness analysis. Behav Res Methods 2008;40:1-7.

-Catania JA, Gibson DR, Chitwood DD, Coates TJ: Methodological problems in AIDS behavioral research: influences on measurement error and participation bias in studies of sexual behavior. Psychol Bull 1990;108:339-362.

-Cherry JC, Moffatt TP, Rodriguez C, Dryden K: Diabetes disease management program for an indigent population empowered by telemedicine technology. Diabetes Technol Ther 2002. 4:783-791.

-Chinman M, Hassell J, Magnabosco J, NowlinFinch N, Marusak S, Young AS: The feasibility of computerized patient self-assessment at mental health clinics. Adm Policy Ment Health 2007;34:401-409.
Chinman M, Young AS, Schell T, Hassell J, Mintz $\mathrm{J}$ : Computer-assisted self-assessment in persons with severe mental illness. J Clin Psychiatry 2004;65:1343-1351.

-Chisolm DJ, Klima J, Gardner W, Kelleher KJ: Adolescent behavioral risk screening and use of health services. Adm Policy Ment Health 2009;36:374-380.

Cumming JJ, Elkins J: Stability of strategy use for addition facts: a training study and implications for instruction. J Cogn Educ 1996;5: 101-116.

Currivan DB, Nyman AL, Turner CF, Biener L: Does telephone audio computer-assisted selfinterviewing improve the accuracy of prevalence estimates of youth smoking? Evidence from the UMass Tobacco Study. Public Opin Q 2004;68:542-564.

Davidson OR, Stevens DE, Goddard GV, Bilkey DK: The performance of a sample of traumatic head-injured patients on some novel computer-assisted neuropsychological tests. Appl Psychol 1987;36:329-342.

Des Jarlais DC, Paone D, Milliken J, Turner CF, Miller H, Gribble J, Friedman SR: Audiocomputer interviewing to measure risk behaviour for HIV among injecting drug users: a quasi-randomised trial. Lancet 1999;353: $1657-1661$

Epstein JF, Barker PR, Kroutil LA: Mode effects in self-reported mental health data. Public Opin Q 2001;65:529-549.

Erdman HP, Klein MH, Greist JH: Direct patient computer interviewing. J Consult Clin Psychol 1985;53:760-773.

Guendelman S, Meade K, Benson M, Chen YQ, Samuels S: Improving asthma outcomes and self-management behaviors of inner-city children: a randomized trial of the Health Buddy interactive device and an asthma diary. Arch Pediatr Adolesc Med 2002;156:114-120.
Günther VK, Schäfer P, Holzner BJ, Kemmler $\mathrm{GW}$ : Long-term improvements in cognitive performance through computer-assisted cognitive training: a pilot study in a residential home for older people. Aging Ment Health 2003;7:200-206.

-Harmon T, Turner CF, Rogers SM, Eggleston E, Roman AM, Villarroel MA, Li S: Impact of T-ACASI on survey measurements of subjective phenomena. Public Opin Q 2009;73:255280.

Islam MM, Topp L, Conigrave KM, van Beek I, Maher L, White A, Day CA: The reliability of sensitive information provided by injecting drug users in a clinical setting: clinician-administered versus audio computer-assisted self-interviewing (ACASI). AIDS Care 2012; 24:1496-1503.

Jamieson LM, Thomson WM, McGee R: An assessment of the validity and reliability of dental self-report items used in a National Child Nutrition Survey. Community Dent Oral Epidemiol 2004;32:49-54.

-Jennings TE, Lucenko BA, Malow RM, Devieux JG: Audio-CASI versus interview method of administration of an HIV/STD risk of exposure screening instrument for teenagers. Int $J$ STD AIDS 2002;13:781-784.

-Johnson AM, Copas AJ, Erens B, Mandalia S, Fenton K, Korovessis C, Field J: Effect of computer-assisted self-interviews on reporting of sexual HIV risk behaviours in a general population sample: a methodological experiment. AIDS 2001;15:111-115.

Kurth AE, Martin DP, Golden MR, Weiss NS, Heagerty PJ, Spielberg F, Holmes KK: A comparison between audio computer-assisted self-interviews and clinician interviews for obtaining the sexual history. Sex Transm Dis 2004;31:719-726. 
Kurth AE, Spielberg F, Rossini A, Group UAW: STD/HIV risk: what should we measure, and how should we measure it? Int J STD AIDS 2001;12(suppl 2):171.

Levin HM: Cost-Effectiveness: A Primer. Beverly Hills, Sage, 1983.

- Metzger DS, Koblin B, Turner C, Navaline H, Valenti F, Holte S, Seage GR 3rd: Randomized controlled trial of audio computer-assisted self-interviewing: utility and acceptability in longitudinal studies. HIVNET Vaccine Preparedness Study Protocol Team. Am J Epidemiol 2000;152:99-106.

- Newman JC, Des Jarlais DC, Turner CF, Gribble J, Cooley P, Paone D: The differential effects of face-to-face and computer interview modes. Am J Public Health 2002;92: 294-297.

- Paperny DM, Aono JY, Lehman RM, Hammar SL, Risser J: Computer-assisted detection and intervention in adolescent high-risk health behaviors. J Pediatr 1990;116:456-462.

Perlis TE, Des Jarlais DC, Friedman SR, Arasteh K, Turner CF: Audio-computerized self-interviewing versus face-to-face interviewing for research data collection at drug abuse treatment programs. Addiction 2004;99:885896.

Robinson R, West R: A comparison of computer and questionnaire methods of history-taking in a genito-urinary clinic. Psychol Health 1992;6:77-84.
Romer D, Hornik R, Stanton B, Black M, Li X, Ricardo I, Feigelman S: 'Talking' computers: a reliable and private method to conduct interviews on sensitive topics with children. J Sex Res 1997;34:3-9.

Schackman BR, Dastur Z, Rubin DS, Berger J, Camhi E, Netherland J, Finkelstein R: Feasibility of using audio computer-assisted selfinterview (ACASI) screening in routine HIV care. AIDS Care 2009;21:992-999.

Schaeffer NC: Asking questions about threatening topics: a selective overview; in Stone AA, Turkkan JS, Bachrach CA, Jobe JB, Kurtzman HS, Cain V (eds): The Science of Self-Report: Implications for Research and Practice. Mahwah, Erlbaum, 2000, pp 105-121.

Schroder KE, Carey MP, Vanable PA: Methodological challenges in research on sexual risk behavior. II. Accuracy of self-reports. Ann Behav Med 2003;26:104-123.

Swadi $\mathrm{H}$ : Validating and improving the validity of self-reports in adolescent substance misuse surveys. J Drug Issues 1990;20:473-486.

Tourangeau R: Remembering what happened: memory errors and survey reports; in Stone AA, Turkkan JS, Bachrach CA, Jobe JB, Kurtzman HS, Cain VS (eds): The Science of Self-Report: Implications for Research and Practice. Mahwah, Erlbaum, 2000, pp 29-47.
Turner CF, Ku L, Rogers SM, Lindberg LD, Pleck JH, Sonenstein FL: Adolescent sexual behavior, drug use, and violence: increased reporting with computer survey technology. Science 1998;280:867-873.

Turner CF, Miller HG, Rogers SM: Survey measurement of sexual behavior: problems and progress; in Bancroft J (ed): Researching Sexual Behavior: Methodological Issues. Bloomington, Indiana University Press, 1997, pp $37-60$.

Webb PM, Zimet GD, Fortenberry JD, Blythe MJ: Comparability of a computer-assisted versus written method for collecting health behavior information from adolescent patients. J Adolesc Health 1999;24:383-388.

Weinhardt LS, Forsyth AD, Carey MP, Jaworski BC, Durant LE: Reliability and validity of selfreport measures of HIV-related sexual behavior: progress since 1990 and recommendations for research and practice. Arch Sex Behav 1998;27:155-180.

-White RF, James KE, Vasterling JJ, Letz R, Marans K, Delaney R, Kraemer HC: Neuropsychological screening for cognitive impairment using computer-assisted tasks. Assessment 2003;10: 86-101.

Williams CA, Templin T, Mosley-Williams AD: Usability of a computer-assisted interview system for the unaided self-entry of patient data in an urban rheumatology clinic. J Am Med Inform Assoc 2004;11:249-259. 